Publisher homepage: www.universepg.com, ISSN: 2663-6913 (Online) \& 2663-6905 (Print)

\title{
Physico-chemical Characterization of Kaptai Lake and Foy's Lake Water Quality Parameters in Chittagong, Bangladesh
}

\author{
Md. Rubel $^{1}{ }^{*}$, Didarul Alam Chowdhury ${ }^{1}$, Mohammad Jamal Uddin Ahmed ${ }^{1}$, and Mohammad Helal \\ Uddin $^{2}$ \\ ${ }^{1}$ Laboratory of Analytical Chemistry, Department of Chemistry, University of Chittagong, Chittagong, Bangladesh; and \\ ${ }^{2}$ Department of Applied Chemistry and Chemical Engineering, University of Chittagong, Chittagong, Bangladesh. \\ *Correspondence: rubelcu90@gmail.com
}

\begin{abstract}
:
In order to protect the quality of environment and human's health, fresh water assets are tremendously important in various ways. To ensure the fresh water resources in Chittagong region of Bangladesh, we have studied the water quality parameters of Kaptai and Foy's Lake. This research has done based on the essential surface water standard parameters such as $\mathrm{pH}$, temperature, DO, BOD, COD, TDS, TSS, TS, EC, hardness, turbidity, salinity, total alkalinity, total acidity, $\mathrm{SO}_{4}{ }^{2-}, \mathrm{PO}_{4}{ }^{3-}, \mathrm{NO}^{3-}-\mathrm{N}, \mathrm{NO}^{2-}, \mathrm{CO}_{2}$, and most of the heavy and toxic metals (As, $\mathrm{Cd}, \mathrm{Cr}, \mathrm{Cu}, \mathrm{Co}, \mathrm{Fe}, \mathrm{Pb}, \mathrm{Mn}, \mathrm{Ni}$, and $\mathrm{Zn}$ ) of two lakes namely Foy's (Chittagong) and Kaptai (Rangamati) Lakes in Chittagong, Bangladesh. The statistical approaches of sampling were utilized for collecting samples. The samples were assembled from ten different locations of each lake. Samples were conserved using satisfactory preservation procedure. Water samples from the surface-water assets were collected from various locations, and tide conditions and at various seasons for continual monitoring during the hydrological years 2014-2015. The results showed that Kaptai Lake, and Foy's Lake all physicochemical parameters are within the permissible limit of WHO guideline. The results also supplied data to view, and quantify the enemy of the impact of climate alter on freshwater resources of this region. The outcomes further showed data for water quality of surface-water resources of greater Chittagong zone to match national and international quality for drinking, agricultural, manufacture and livestock requirements. A strategic water quality management plan has been proposed.
\end{abstract}

Keywords: Physicochemical assessment, Water quality, Kaptai Lake, Characterization, and Climate change.

\section{INTRODUCTION:}

Lakes and surface water bodies are considered as one of the planet's most significant freshwater assets and provide innumerable interests. Lakes are inert surface water bodies, receive and storages rain fall water. Increased anthropogenic pursuit in and around the water bodies destroy the aquatic ecosystems and eventually the physico-chemical properties of water (Upadhyay et al., 2010). The Research is to assess the quality and quantity of the earth assets of the nation, and to provide information that will aid resource managers and policy makers at national, regional and local levels in producing sound decisions. Water quality refers to the chemical, physical and biological characteristics of water (Diersing, 2009; Lasat, 2002). Chemical assessment of water quality status and trends is a significant part of this study. One of the greatest challenges faced by water assets scientists is 
acquiring easy information that will guide the use, and shelter of the nation's water resources (WHO, 2004). That challenge is being represented by national, divisional, inter-district and local water resource agencies, and by large academic institutions (Hirsch, 2007). These institutions are collecting water standard data for a host of purposes that include compliance with authorizes and water supply standards; development of remediation plans for desire contamination problem; operational decisions on industrial, wastewater, or waste water supply facilities; and study on factors that affect water quality. An additional require for standard quality information is to provide a basis on which regional, and native level policy resolve can be based (Ahmed et al., 2018). Foy's Lake is one of the major significant man-made lakes in Chittagong, Bangladesh that was built by building a dam over the stream that decline from the slopes in the northern region of Chittagong in 1924. Intelligent decisions must be based on stable information (Hirsch, 2007). Because Bangladesh is fortunate enough having an extensive and huge water resources scattered all over the country in the form of small pond, beels (natural depression), lakes, canals, small and large rivers and estuaries covering an area of about 4.34 million hectares. In Bengali, Bangladesh is called as 'nodimatrik desh'. This is indeed literally true because rivers gave birth to this land.

Bangladesh is located in the low-lying GangesBrahmaputra River Delta, one of the greatest deltas in the world, is criss-crossed by different rivers, their tributaries and distributaries. Straddling the Tropic of Cancer prevalence, Bangladeshi environment is tropical with a mild winter from month of October to March, a dry, humid summer from the month of March to June. A hot and humid monsoon season lasts from the month of June to October, and distributes most of the country's rainfall. The hydroponic creating media allows sufficient root distribution of plants, and maintains homogeneous state of growing media, which is restricted in the pot plant experiments (Hossain et al., 2019; Salt et al., 1995). Now a critical need exists for accurate facts and rationale concept to provide approach for organizing our affairs. Pollution from human and industrial sources is the main water quality problem in the river systems near urban areas of Dhaka (WHO, 1991). However, any comprehensive assessment of Chittagong division's surface water quality parameters has yet to be completed. Some trace metals such as $\mathrm{Cu}, \mathrm{Fe}, \mathrm{Mn}, \mathrm{Zn}$, etc., at catalytic numbers as metalloenzymes and as cofactors of enzymes, are necessary to living organisms for their usual physiological activities. But the high concentration of $\mathrm{Cu}$ has been associates with liver damage, and $\mathrm{Zn}$ may create harmful nutrient interactions with $\mathrm{Cu}$ (Majagi et al., 2008).

Chittagong is generally called the business capital of Bangladesh. The challenges posed by climate alter, and growing economic improvement requires that the quantity and quality of water assets in Bangladesh be regulated by sustainable development policies. Without such an idea, Bangladesh will continue to face vital challenges to gain economic growth to help the growing population confined in a denselypopulated land with reducing water resources (Ahmed and Haque, 2010). At present, Kaptai Lake assists poor-scale fisheries, which is enrich in fish species diversity, and contributing approximately 8980 metric ton freshwater fish annually (FRSS, 2012). The main objectives of the study is to know the present status of physico-chemical parameters of water of the 2 selected lakes in Chittagong, to assess their interrelationship and to compare the present results with results obtained previously in the lakes of Chittagong as well as other lakes in home and abroad.

\section{MATERIALS AND METHODS:}

Sample Collection - A survey work was conducted fiscal year 2014-2016 for identifying sampling spots and the current quality conditions for a large part of Kaptai Lake and Foy's Lake in greater Chittagong region. The surface water samples were collected in the boat if possible in the middle of the flow. Two to four sub samples of equal volume were collected from vertical section. The water samples were collected within 3-9 inches from the surface of the water. Surface samples from different points of greater Chittagong district were collected for this study. Samples were piled up in amber color polyethylene bottle washed by rinsing thoroughly with $8 \mathrm{M} \mathrm{HNO}_{3}$, followed by repeated washing with distilled water. The samples were mixed well and a sample of 1.0-1.5$\mathrm{L}$ was transferred-for analysis in the laboratory. 
Apparatus - A Shimadzu (Kyoto, Japan) (Model1800PC) double-beam UV/VIS spectrophotometer and a Jenway (England, UK) (Model-3010) pH meter with a union of electrodes were used for determines of the absorbance and $\mathrm{pH}$, respectively. A Varian ModelAA240FS quick sequential atomic absorption spectrophotometer (AAS) was used for studies of trace elements in water using a respective wavelength and hollow cathode lamp.

Sample Processing and Analysis - A glass thermometer was used for the measurement of temperature. $\mathrm{pH}, \mathrm{EC}$ and TDS were studied at the sampling site by Hanna Combo meter, Model HI 98129. TS and TSS were studied by drying and weighing approach. Measurement of DO was done by Jenway 970 DO meter at the sampling area. BOD was studied from the differences of preliminary and 5 day, DO by DO meter. COD was estimated by titrimetric approach after 2 hours open reflux. Acidity, and dissolved carbon dioxide were studied by titrimetric approach using standard $0.02 \mathrm{M} \mathrm{NaOH}$. Total and phenolphthalein alkalinity were studied by titrimetric approach using phenolphthalein, and methyl orange as indicators.

Hardness was studied by complex metric titration approach. Dissolved hydrogen sulphide (DHS) was studied by iodimetric approach using standard iodine solution (WHO, 1991). Ammonia-N was studied by colorimetric nesslerization approach after distillation of collected samples. Total nitrate and nitrite-N was studied by colorimetric nesslerization procedure after reducing samples by Deverda's alloy (WHO, 1984). Sulphate $\mathrm{S}$ was studied by colorimetric barium chloride turbidimetric approach. Nitrite-N was studied by colorimetric method after formation of a reddish purple azo dye produced at $\mathrm{pH} 2.0$ to 2.5 by coupling diazotized sulfanilamide with $\mathrm{N}$ - (1-naphthyl)ethylene diaminedi- hydrochloride (NED hydrochloride). Nitrate-N was estimated by colorimetric method in the ultraviolet range. Iron was studied by colorimetric method using 1, 10phenanthroline as chelating agent. Manganese was studied by colorimetric per-sulfate oxidation method. In colorimetric methods Zinc, copper, lead, cadmium, cobalt, nickel and chromium were studied by the measure of metals by UV-Visible and atomic absorption spectrophotometry samples were digested according to the wet-digestion method (EWD, 2009).

In colorimetric techniques a Shimadzu, Model-1800 UV-Vis spectrophotometer was used. Zinc, copper, lead, cadmium, cobalt, nickel and chromium were studied by Varian Model-AA240FS fast sequential atomic absorption spectrophotometer (AAS). For the determination of metals by UV-Visible Spectrophotometry, and atomic absorption spectrophotometry samples were digested as stated by to the wet-digestion method (Flanagan et al., 2001).

Table 1: The parameters studied during the analysis and processing of water samples.

\begin{tabular}{|l|l|l|l|}
\hline Parameters & Units & Parameters & Units \\
\hline Temperature & ${ }^{0} \mathrm{C}$ & Chloride $\left(\mathrm{Cl}^{-}\right)$ & $\mathrm{mgL}^{-1}$ \\
\hline $\mathrm{pH}$ & - & Sulphate $\left(\mathrm{SO}_{4}^{2-}\right)$ & $\mathrm{mgL}^{-1}$ \\
\hline TDS & $\mathrm{mgL}^{-1}$ & Phosphate $\left(\mathrm{PO}_{4}^{3-}\right)$ & $\mathrm{mgL}^{-1}$ \\
\hline EC & $\mu \mathrm{Scm}^{-1}$ & Nitrate $\left(\mathrm{NO}_{3}^{-}-\mathrm{N}\right)$ & $\mathrm{mgL}^{-1}$ \\
\hline TSS & $\mathrm{mgL}^{-1}$ & Nitrite $\left(\mathrm{NO}_{2}^{-}-\mathrm{N}\right)$ & $\mathrm{mgL}^{-1}$ \\
\hline TS & $\mathrm{mgL}^{-1}$ & Arsenic $(\mathrm{As})$ & $\mathrm{mgL}^{-1}$ \\
\hline DO & $\mathrm{mgL}^{-1}$ & Cadmium $(\mathrm{Cd})$ & $\mathrm{mgL}^{-1}$ \\
\hline BOD & $\mathrm{mgL}^{-1}$ & Cromium $(\mathrm{Cr})$ & $\mathrm{mgL}^{-1}$ \\
\hline COD & $\mathrm{mgL}^{-1}$ & Copper $(\mathrm{Cu})$ & $\mathrm{mgL}^{-1}$ \\
\hline Turbidity & $\mathrm{NTU}^{-1}$ & Cobalt $(\mathrm{Co})$ & $\mathrm{mgL}^{-1}$ \\
\hline Salinity & $\mathrm{mgL}^{-1}$ & Iron $(\mathrm{Fe})$ & $\mathrm{mgL}^{-1}$ \\
\hline Total Hardness & $\mathrm{mgL}^{-1}$ & Lead $(\mathrm{Pb})$ & $\mathrm{mgL}^{-1}$ \\
\hline Total Alkalinity & $\mathrm{mgL}^{-1}$ & Manganese $(\mathrm{Mn})$ & $\mathrm{mgL}^{-1}$ \\
\hline Total Acidity & $\mathrm{mgL}^{-1}$ & Nickel $(\mathrm{Ni})$ & $\mathrm{mgL}^{-1}$ \\
\hline CO & $\mathrm{mgL}_{2}^{-1}$ & Zinc $(\mathrm{Zn})$ & $\mathrm{mgL}^{-1}$ \\
\hline
\end{tabular}


RESULTS AND DISCUSSION:

The present physico-chemical study of Kaptai and Foy's Lake water resources of greater Chittagong region discloses the status of water quality of these two lakes. EC (95.6-115.20 $\left.\mu \mathrm{Scm}^{-1}\right)$, and TDS (47.80- $\left.57.600 \mathrm{mgL}^{-1}\right)$. DO was found (7.60-8.20 $\left.\mathrm{mgL}^{-1}\right)$. The result of physico-chemical analyzes of Kaptai and Foy's Lake fresh water of the studied sites are presented in the below Tables.

Table 2: Surface water quality of Kaptai Lake at different locations.

\begin{tabular}{|c|c|c|c|c|c|c|}
\hline \multirow[b]{2}{*}{ Parameters } & \multirow[b]{2}{*}{ Units } & \multicolumn{5}{|c|}{ Locations } \\
\hline & & 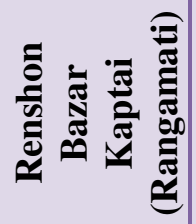 & 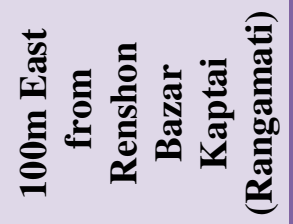 & 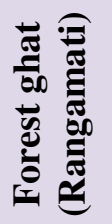 & 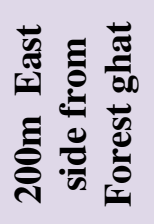 & 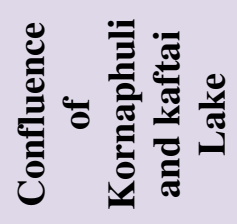 \\
\hline Temperature & ${ }^{0} \mathrm{C}$ & 29.5 & 29.8 & 30.0 & 29.7 & 30.3 \\
\hline $\mathrm{pH}$ & - & 8.8 & 8.4 & 8.5 & 8.2 & 8.0 \\
\hline TDS & $\mathrm{mgL}^{-1}$ & 57.6 & 54 & 50 & 49 & 48.8 \\
\hline $\mathrm{EC}$ & $\mu \mathrm{Scm}^{-1}$ & 115.2 & 107 & 100.1 & 99 & 97.6 \\
\hline TSS & $\mathrm{mgL}^{-1}$ & 2 & 2.6 & 3.0 & 4.2 & 4.4 \\
\hline $\mathrm{TS}$ & $\mathrm{mgL}^{-1}$ & 59.6 & 56.6 & 53 & 53.2 & 51.2 \\
\hline DO & $\mathrm{mgL}^{-1}$ & 8.1 & 8.0 & 8.2 & 7.7 & 8.0 \\
\hline BOD & $\mathrm{mgL}^{-1}$ & 0.3 & 0.4 & 0.5 & 0.4 & 0.2 \\
\hline COD & $\mathrm{mgL}^{-1}$ & 38 & 43 & 50 & 45 & 25 \\
\hline Turbidity & NTU & 1 & 1.3 & 1.5 & 2.1 & 2.2 \\
\hline Salinity & $\mathrm{mgL}^{-1}$ & 0.02 & 0.04 & 0.03 & 0.06 & 0.03 \\
\hline T. Hardness & $\mathrm{mgL}^{-1}$ & 42 & 38 & 36 & 35 & 40 \\
\hline T. Alkalinity & $\mathrm{mgL}^{-1}$ & 44.5 & 46.0 & 45.23 & 47.82 & 42.90 \\
\hline T.Acidity & $\mathrm{mgL}^{-1}$ & 10 & 11 & 10.5 & 10.2 & 9.5 \\
\hline $\mathrm{CO}_{2}$ & $\mathrm{mgL}^{-1}$ & 6.0 & 6.5 & 6.7 & 8.0 & 7.0 \\
\hline Chloride $\left(\mathrm{Cl}^{-}\right)$ & $\mathrm{mgL}^{-1}$ & 6 & 4 & 2 & 2 & 4 \\
\hline Sulphate $\left(\mathrm{SO}_{4}{ }^{2-}\right)$ & $\mathrm{mgL}^{-1}$ & 3.0 & 1.0 & 2.0 & 1.3 & 2.1 \\
\hline Phosphate $\left(\mathrm{PO}_{4}^{3-}\right)$ & $\mathrm{mgL}^{-1}$ & 0.01 & 0.03 & 0.02 & 0.01 & 0.01 \\
\hline Nitrate $\left(\mathrm{NO}_{3}^{-}-\mathrm{N}\right)$ & $\mathrm{mgL}^{-1}$ & 1.26 & 0.80 & 0.50 & 1.1 & 1.5 \\
\hline Nitrite $\left(\mathrm{NO}_{2}^{-}-\mathrm{N}\right)$ & $\mathrm{mgL}^{-1}$ & 5.67 & 3.6 & 2.25 & 4.95 & 6.75 \\
\hline Arsenic (As) & $\mathrm{mgL}^{-1}$ & BDL & 0.02 & BDL & 0.03 & BDL \\
\hline Cadmium (Cd) & $\mathrm{mgL}^{-1}$ & BDL & BDL & BDL & BDL & BDL \\
\hline Cromium (Cr) & $\mathrm{mgL}^{-1}$ & 0.03 & 0.02 & BDL & BDL & 0.04 \\
\hline 2Copper $(\mathrm{Cu})$ & $\mathrm{mgL}^{-1}$ & O.1 & 0.3 & 0.2 & 0.4 & BDL \\
\hline Cobalt (Co) & $\mathrm{mgL}^{-1}$ & BDL & BDL & BDL & BDL & BDL \\
\hline Iron $(\mathrm{Fe})$ & $\mathrm{mgL}^{-1}$ & 0.1 & 0.2 & 0.3 & 1.2 & 1.3 \\
\hline Lead $(\mathrm{Pb})$ & $\mathrm{mgL}^{-1}$ & BDL & BDL & BDL & BDL & BDL \\
\hline Manganese (Mn) & $\mathrm{mgL}^{-1}$ & 0.04 & 0.06 & 0.03 & 0.07 & BDL \\
\hline Nickel (Ni) & $\mathrm{mgL}^{-1}$ & BDL & BDL & BDL & BDL & BDL \\
\hline Zinc $(\mathrm{Zn})$ & $\mathrm{mgL}^{-1}$ & 1.2 & 1.4 & 1.6 & 1.1 & BDL \\
\hline
\end{tabular}

Where; $\mathrm{m}=$ Meter 
Different water quality parameters of the Kaptai Lake studied (Table 2, $3 \&$ \& ) show that $\mathrm{pH}$ was found in the alkaline range (7.7-8.8) and water was found in acidity (2.8-4.60 $\left.\mathrm{mgL}^{-1}\right)$. Alkalinity of the Kaptai Lake (42.90-51.00 $\mathrm{mgL}^{-1}$ ) is mainly for carbonates and bicarbonates as phenolphthalein alkalinity was not detected in any of the samples. Water was found in turbidity as (1.00-2.5 NTU). Some parameters contributed to the changes of the groundwater geochemistry behavior discuss the effect of rising isolation of freshwater from the aquifer (Aris et al., 2007).

Table 3: Surface water quality of Kaptai Lake at different locations.

\begin{tabular}{|c|c|c|c|c|c|c|}
\hline \multirow[b]{2}{*}{ Parameters } & \multirow[b]{2}{*}{ Units } & \multicolumn{5}{|c|}{ Locations } \\
\hline & & 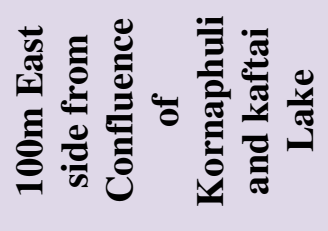 & 晃 & 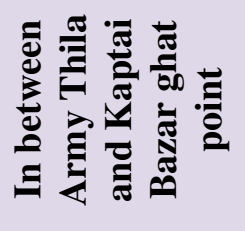 & 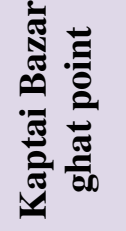 & 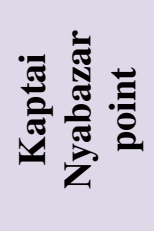 \\
\hline Temperature & ${ }^{0} \mathrm{C}$ & 30.2 & 29.9 & 29.8 & 29.6 & 30.1 \\
\hline $\mathrm{pH}$ & - & 7.8 & 7.7 & 8.0 & 8.1 & 8.3 \\
\hline TDS & $\mathrm{mgL}^{-1}$ & 49.5 & 47.8 & 51 & 52 & 49.9 \\
\hline $\mathrm{EC}$ & $\mu \mathrm{Scm}^{-1}$ & 98 & 95.6 & 101 & 104 & 102 \\
\hline TSS & $\mathrm{mgL}^{-1}$ & 5.0 & 3.2 & 4.4 & 3.4 & 2.4 \\
\hline TS & $\mathrm{mgL}^{-1}$ & 54.5 & 51 & 55.4 & $55^{4}$ & 52.3 \\
\hline DO & $\mathrm{mgL}^{-1}$ & 8.1 & 7.9 & 8.0 & 7.8 & 7.6 \\
\hline BOD & $\mathrm{mgL}^{-1}$ & 0.2 & 0.4 & 0.2 & 0.5 & 0.3 \\
\hline COD & $\mathrm{mgL}^{-1}$ & 40 & 52 & 35 & 55 & 47 \\
\hline Turbidity & NTU & 2.5 & 1.6 & 2.2 & 1.7 & 1.2 \\
\hline Salinity & $\mathrm{mgL}^{-1}$ & 0.05 & 0.04 & 0.06 & 0.08 & 0.05 \\
\hline T. Hardness & $\mathrm{mgL}^{-1}$ & 37 & 39 & 41 & 41.5 & 36.5 \\
\hline T. Alkalinity & $\mathrm{mgL}^{-1}$ & 48.25 & 47.0 & 49.80 & 51.0 & 45.89 \\
\hline T. Acidity & $\mathrm{mgL}^{-1}$ & 9.0 & 8.8 & 9.4 & 9.5 & 12 \\
\hline $\mathrm{CO}_{2}$ & $\mathrm{mgL}^{-1}$ & 7.5 & 6.8 & 8.3 & 7.6 & 9.5 \\
\hline Chloride $\left(\mathrm{Cl}^{-}\right)$ & $\mathrm{mgL}^{-1}$ & 10 & 4 & 6 & 8 & 4 \\
\hline Sulphate $\left(\mathrm{SO}_{4}{ }^{2-}\right)$ & $\mathrm{mgL}^{-1}$ & 2.3 & 1.1 & 2.9 & 1.2 & 2.0 \\
\hline Phosphate $\left(\mathrm{PO}_{4}{ }^{3-}\right)$ & $\mathrm{mgL}^{-1}$ & 0.02 & 0.03 & 0.02 & 0.05 & 0.03 \\
\hline Nitrate $\left(\mathrm{NO}_{3}^{-}-\mathrm{N}\right)$ & $\mathrm{mgL}^{-1}$ & 1.20 & 0.90 & 0.70 & 1.17 & 1.3 \\
\hline Nitrite $\left(\mathrm{NO}_{2}^{-}-\mathrm{N}\right)$ & $\mathrm{mgL}^{-1}$ & 5.4 & 4.05 & 3.15 & 5.26 & 5.85 \\
\hline Arsenic (As) & $\mathrm{mgL}^{-1}$ & BDL & BDL & 0.03 & 0.01 & BDL \\
\hline Cadmium $(\mathrm{Cd})$ & $\mathrm{mgL}^{-1}$ & BDL & BDL & BDL & BDL & BDL \\
\hline Chromium (Cr) & $\mathrm{mgL}^{-1}$ & BDL & 0.03 & BDL & 0.04 & BDL \\
\hline Copper $(\mathrm{Cu})$ & $\mathrm{mgL}^{-1}$ & 0.5 & 0.2 & 0.7 & 0.1 & 0.2 \\
\hline Cobalt (Co) & $\mathrm{mgL}^{-1}$ & BDL & BDL & BDL & BDL & BDL \\
\hline Iron $(\mathrm{Fe})$ & $\mathrm{mgL}^{-1}$ & 0.9 & 1.6 & 1.4 & 1.3 & 1.7 \\
\hline Lead $(\mathrm{Pb})$ & $\mathrm{mgL}^{-1}$ & BDL & BDL & BDL & BDL & BDL \\
\hline Manganese (Mn) & $\mathrm{mgL}^{-1}$ & BDL & 0.02 & 0.04 & BDL & 0.08 \\
\hline Nickel (Ni) & $\mathrm{mgL}^{-1}$ & BDL & BDL & BDL & BDL & BDL \\
\hline Zinc (Zn) & $\mathrm{mgL}^{-1}$ & 1.5 & 1.8 & 1.7 & 2.1 & 1.9 \\
\hline
\end{tabular}

Where; $\mathrm{m}=$ Meter 
Total hardness was found in the range (35.00-42.00 $\left.\mathrm{mgL}^{-1}\right)$. Chloride (7.00-12.00 $\left.\mathrm{mgL}^{-1} 1\right)$, BOD $(0.2-0.5$ $\left.\mathrm{mgL}^{-1}\right)$, nitrite- $\mathrm{N}(2.25-6.75)$, and nitrate-N $(0.70-1.3$ mgL-1). Phosphate-P (0.010-0.052 $\left.\mathrm{mgL}^{-1}\right)$ and sulfate$\mathrm{S}$ (1.1-3.00 $\left.\mathrm{mgL}^{-1}\right)$ were also found within the permissible limit. Iron $\left(0.1-1.7 \mathrm{mg} \mathrm{L}^{-1}\right)$ and manganese $\left(0.00-0.07 \mathrm{mg} \mathrm{L}^{-1}\right)$ were found within the limit. No significant variation was observed in water quality among the ten points. The F-factor was developed in a situation when the acidification was increasing and it has also been a basis for calculating critical loads of acidity, e.g. the Steady-State Water Chemistry model (Henriksen and Posch, 2001).

Table 4: Surface water quality of Foy's lake at different locations.

\begin{tabular}{|c|c|c|c|c|c|c|}
\hline \multirow[b]{2}{*}{ Parameters } & \multirow[b]{2}{*}{ Units } & \multicolumn{5}{|c|}{ Locations } \\
\hline & & 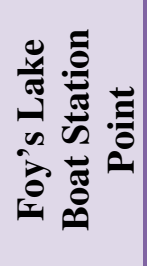 & 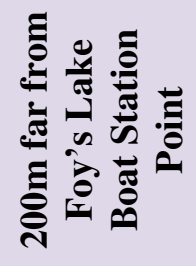 & 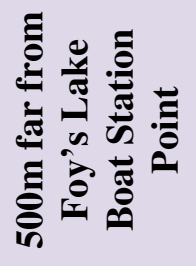 & 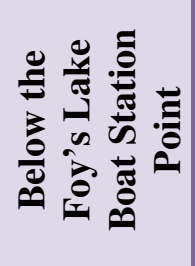 & 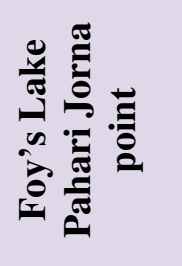 \\
\hline Temperature & ${ }^{0} \mathrm{C}$ & 29.0 & 30.1 & 29.9 & 30.0 & 30.3 \\
\hline $\mathrm{pH}$ & - & 7.5 & 7.7 & 8.0 & 7.6 & 7.9 \\
\hline TDS & $\mathrm{mgL}^{-1}$ & 33.5 & 34 & 33 & 35.5 & 36.4 \\
\hline $\mathrm{EC}$ & $\mu \mathrm{Scm}^{-1}$ & 67 & 68 & 66 & 71 & 72.8 \\
\hline TSS & $\mathrm{mgL}^{-1}$ & 3.2 & 4.6 & 5.0 & 3.8 & 4.8 \\
\hline TS & $\mathrm{mgL}^{-1}$ & 36.7 & 38.6 & 38.0 & 39.3 & 41.2 \\
\hline DO & $\mathrm{mgL}^{-1}$ & 8.1 & 7.7 & 7.9 & 8.2 & 7.8 \\
\hline BOD & $\mathrm{mgL}^{-1}$ & 1.5 & 0.8 & 0.2 & 1.7 & 0.6 \\
\hline COD & $\mathrm{mgL}^{-1}$ & 63 & 45 & 28 & 70 & 38 \\
\hline Turbidity & NTU & 1.6 & 2.3 & 2.5 & 1.9 & 2.4 \\
\hline Salinity & $\mathrm{mgL}^{-1}$ & 0.06 & 0.08 & 0.05 & 0.07 & 0.04 \\
\hline T. Hardness & $\mathrm{mgL}^{-1}$ & 26 & 27 & 24 & 25.5 & 28.8 \\
\hline T. Alkalinity & $\mathrm{mgL}^{-1}$ & 32 & 34 & 30 & 31.5 & 32.5 \\
\hline T. Acidity & $\mathrm{mgL}^{-1}$ & 10.01 & 14.80 & 10.24 & 18.27 & 20.19 \\
\hline $\mathrm{CO}_{2}$ & $\mathrm{mgL}^{-1}$ & 4 & 8 & 6 & 16 & 16 \\
\hline Chloride $\left(\mathrm{Cl}^{-}\right)$ & $\mathrm{mgL}^{-1}$ & 2 & 6 & 4 & 8 & 10 \\
\hline Sulphate $\left(\mathrm{SO}_{4}{ }^{2-}\right)$ & $\mathrm{mgL}^{-1}$ & 6 & 8 & 10 & 7.5 & 12 \\
\hline Phosphate $\left(\mathrm{PO}_{4}^{3-}\right)$ & $\mathrm{mgL}^{-1}$ & 10 & 9.8 & 8.0 & 10.5 & 11 \\
\hline Nitrate $\left(\mathrm{NO}_{3}{ }^{-}-\mathrm{N}\right)$ & $\mathrm{mgL}^{-1}$ & 3 & 6 & 7 & 7.5 & 8 \\
\hline Nitrite $\left(\mathrm{NO}_{2}^{-}-\mathrm{N}\right)$ & $\mathrm{mgL}^{-1}$ & 0.01 & 0.21 & 0.32 & 0.30 & 0.41 \\
\hline Arsenic (As) & $\mathrm{mgL}^{-1}$ & $\mathrm{BDL}$ & BDL & 0.02 & 0.03 & BDL \\
\hline Cadmium (Cd) & $\mathrm{mgL}^{-1}$ & BDL & BDL & BDL & BDL & BDL \\
\hline Chromium (Cr) & $\mathrm{mgL}^{-1}$ & BDL & BDL & BDL & 0.03 & 0.04 \\
\hline Copper $(\mathrm{Cu})$ & $\mathrm{mgL}^{-1}$ & 0.1 & BDL & 0.14 & 0.16 & BDL \\
\hline Cobalt (Co) & $\mathrm{mgL}^{-1}$ & BDL & 0.224 & BDL & BDL & BDL \\
\hline Iron $(\mathrm{Fe})$ & $\mathrm{mgL}^{-1}$ & 0.23 & 0.16 & 0.18 & 0.32 & 0.28 \\
\hline Lead $(\mathrm{Pb})$ & $\mathrm{mgL}^{-1}$ & BDL & BDL & BDL & BDL & BDL \\
\hline Manganese (Mn) & $\mathrm{mgL}^{-1}$ & $\mathrm{BDL}$ & 0.5 & 0.63 & BDL & 0.74 \\
\hline Nickel (Ni) & $\mathrm{mgL}^{-1}$ & BDL & BDL & BDL & 0.6 & BDL \\
\hline Zinc (Zn) & $\mathrm{mgL}^{-1}$ & 4.05 & 3.15 & 2.25 & 3.6 & 2.7 \\
\hline
\end{tabular}

Where; $\mathrm{m}=$ Meter 
Different water quality parameters of the Foy's Lake studied (Table $4 \&$ 6) show that $\mathrm{pH}$ was found in the alkaline range (7.5-8.0) and water was found in acidity (8.00-14.00 $\left.\mathrm{mgL}^{-1}\right)$. EC (66-72.80 $\left.\mu \mathrm{Scm}^{-1}\right)$, and TDS (33.00-72.80 $\left.\mathrm{mLL}^{-1}\right)$. DO was found $\left(7.70-8.10 \mathrm{mgL}^{-}\right.$ $\left.{ }^{1}\right)$. Across literature there are strong discrepancies regarding species specific sensitivity towards copper. For example, experiments with Daphnia magna claim 48-h EC50 of 7.7-430 $\mu \mathrm{g} \mathrm{Cu/l}$ and 48-h LC50 of 5-86 $\mu \mathrm{g} \mathrm{Cu} / \mathrm{l}$ (Kramer et al., 2004).

Table 5: Comparative study of water quality parameters of the Kaptai Lake.

\begin{tabular}{|c|c|c|c|c|c|c|}
\hline Parameters & Units & Min & Max & Mean & BSTI & WHO \\
\hline Temperature & ${ }^{0} \mathrm{C}$ & 29.5 & 30.2 & $29.89 \pm 0.24 *$ & $20-30$ & $20-30$ \\
\hline $\mathrm{pH}$ & - & 7.7 & 8.8 & $8.18 \pm 0.38$ & $6.4-7.4$ & $6.5-8.5$ \\
\hline TDS & $\mathrm{mgL}^{-1}$ & 47.8 & 57.6 & $50.96 \pm 1.10$ & Max-500 & Max-1000 \\
\hline $\mathrm{EC}$ & $\mu \mathrm{Scm}^{-1}$ & 95.6 & 115.2 & $101.95 \pm 3.43$ & - & - \\
\hline TSS & $\mathrm{mgL}^{-1}$ & 2 & 5.0 & $3.46 \pm 1.05$ & - & - \\
\hline $\mathrm{TS}$ & $\mathrm{mgL}^{-1}$ & 51 & 59.6 & $54.22 \pm 3.01$ & & \\
\hline DO & $\mathrm{mgL}^{-1}$ & 7.6 & 8.2 & $7.94 \pm 0.21$ & Mini-6 & Max-6.5-8.5 \\
\hline BOD & $\mathrm{mgL}^{-1}$ & 0.2 & 0.5 & $0.34 \pm 0.10$ & - & \\
\hline COD & $\mathrm{mgL}^{-1}$ & 46 & 148 & $43 \pm 5.12$ & - & - \\
\hline Turbidity & NTU & 1 & 2.5 & $1.73 \pm 0.52$ & Max-5 & Max-5 \\
\hline Salinity & $\mathrm{mgL}^{-1}$ & 0.02 & 0.06 & $0.04 \pm 0.01$ & - & - \\
\hline T. Hardness & $\mathrm{mgL}^{-1}$ & 35 & 42 & $38.6 \pm 2.45$ & Max-500 & Max-500 \\
\hline T. Alkalinity & $\mathrm{mgL}^{-1}$ & 42.9 & 51 & $46.83 \pm 2.83$ & - & - \\
\hline T. Acidity & $\mathrm{mgL}^{-1}$ & 8.8 & 14 & $10.29 \pm 1.82$ & - & - \\
\hline $\mathrm{CO}_{2}$ & $\mathrm{mgL}^{-1}$ & 6.0 & 8.3 & $7.39 \pm 0.80$ & - & - \\
\hline Chloride $\left(\mathrm{Cl}^{-}\right)$ & $\mathrm{mgL}^{-1}$ & 4.00 & 6.00 & $5.10 \pm 1.05$ & Max-500 & Max-250 \\
\hline Sulphate $\left(\mathrm{SO}_{4}{ }^{2-}\right)$ & $\mathrm{mgL}^{-1}$ & 1.1 & 3.0 & $1.89 \pm 0.66$ & Max-400 & Max-400 \\
\hline Phosphate $\left(\mathrm{PO}_{4}^{3-}\right)$ & $\mathrm{mgL}^{-1}$ & 0.010 & 0.052 & $0.02 \pm 0.004$ & Max-6 & - \\
\hline Nitrate $\left(\mathrm{NO}_{3}^{-}-\mathrm{N}\right)$ & $\mathrm{mgL}^{-1}$ & 0.70 & 1.3 & $1.043 \pm 0.210$ & Max-4.5 & Max-10 \\
\hline Nitrite $\left(\mathrm{NO}_{2}^{-}-\mathrm{N}\right)$ & $\mathrm{mgL}^{-1}$ & 2.25 & 6.75 & $4.69 \pm 1.56$ & Nil & 0.50 \\
\hline Arsenic (As) & $\mathrm{mgL}^{-1}$ & 0.00 & 0.03 & $0.01 \pm 0.00$ & 0.05 & 0.01 \\
\hline Cadmium $(\mathrm{Cd})$ & $\mathrm{mgL}^{-1}$ & 0.00 & 0.00 & $0.00 \pm 0.00$ & 0.005 & 0.003 \\
\hline Chromium (Cr) & $\mathrm{mgL}^{-1}$ & 0.00 & 0.03 & $0.002 \pm 0.001$ & 0.05 & 0.05 \\
\hline Copper $(\mathrm{Cu})$ & $\mathrm{mgL}^{-1}$ & 0.00 & 0.7 & $0.46 \pm 0.25$ & 1.0 & 0.05 \\
\hline Cobalt (Co) & $\mathrm{mgL}^{-1}$ & 0.00 & 0.00 & $0.00 \pm 0.00$ & 0.003 & 0.005 \\
\hline Iron $(\mathrm{Fe})$ & $\mathrm{mgL}^{-1}$ & 0.1 & 1.7 & $1.23 \pm 0.87$ & $0.3-1.0$ & 0.3 \\
\hline Lead $(\mathrm{Pb})$ & $\mathrm{mgL}^{-1}$ & 0.00 & 0.00 & $0.00 \pm 0.00$ & Max-0.1 & Max-0.1 \\
\hline Manganese (Mn) & $\mathrm{mgL}^{-1}$ & 0.00 & 0.07 & $0.03 \pm 0.02$ & 0.01 & 1.5 \\
\hline Nickel (Ni) & $\mathrm{mgL}^{-1}$ & 0.00 & 0.00 & $0.00 \pm 0.00$ & $0.3-1.0$ & $0.1-1.0$ \\
\hline Zinc $(\mathrm{Zn})$ & $\mathrm{mgL}^{-1}$ & 0.00 & 2.1 & $1.98 \pm 0.98$ & 5.0 & 3.0 \\
\hline
\end{tabular}

Note: Min $=$ Minimum, Max $=$ Maximum. BSTI $=$ Bangladesh Standards and Testing Institute. WHO = World Health Organization. *= The measure of precision is the standard deviation (s).

Alkalinity of the Foy's Lake $\left(20.80-25.19 \mathrm{mgL}^{-1}\right)$ is mainly for carbonates and bicarbonates as phenolphthalein alkalinity was not detected in any of the samples. Water was found in turbidity as $(5.00-$ $7.00 \mathrm{NTU})$. Total hardness was found in the range (30.00-32.50 $\mathrm{mLL}^{-1}$ ). Chloride (4.00-8.00 $\mathrm{mgL}^{-1}$ ),
BOD (0.2-1.7 $\left.\mathrm{mgL}^{-1}\right)$, nitrite-N (2.25-4.05), and nitrate-N (0.50 - $\left.0.9 \mathrm{mgL}^{-1}\right)$. Phosphate-P (0.01 - 0.51 $\left.\mathrm{mgL}^{-1}\right)$ and sulfate-S (3.0 - $\left.8.00 \mathrm{mgL}^{-1}\right)$ were also found within the permissible limit. Iron $(0.16-0.32 \mathrm{mg}$ $\left.\mathrm{L}^{-1}\right)$ and manganese $\left(0.00-0.74 \mathrm{mgL}^{-1}\right)$ were found within the limit. 
No significant variation was observed in water quality among the ten points. Similar result was observed (Kabir and Naser, 2011) where they reported alkaline water in Chand bill Baor Oxbow Lake, Bangladesh and also described alkaline nature of Maheshara Lake water in Gorakhpur, India (Shukla et al., 2013).

Table 6: Comparative study of water quality parameters of the Foy's Lake.

\begin{tabular}{|c|c|c|c|c|c|c|}
\hline Parameters & Units & Min & Max & Mean & BSTI & WHO \\
\hline Temperature & ${ }^{0} \mathrm{C}$ & 29 & 30.3 & $29.86 \pm 0.24 *$ & $20-30$ & $20-30$ \\
\hline $\mathrm{pH}$ & - & 7.5 & 8.0 & $7.74 \pm 0.17$ & $6.4-7.4$ & $6.5-8.5$ \\
\hline TDS & $\mathrm{mgL}^{-1}$ & 33 & 36.4 & $34.48 \pm 1.91$ & Max-500 & Max-1000 \\
\hline $\mathrm{EC}$ & $\mu \mathrm{Scm}^{-1}$ & 66 & 72.8 & $68.96 \pm 2.38$ & - & - \\
\hline TSS & $\mathrm{mgL}^{-1}$ & 10 & 14 & $12 \pm 1.40$ & - & - \\
\hline TS & $\mathrm{mgL}^{-1}$ & 43.5 & 49.5 & $46.48 \pm 2.10$ & & \\
\hline DO & $\mathrm{mgL}^{-1}$ & 7.7 & 8.1 & $7.94 \pm 0.14$ & Mini-6 & Max-6.5-8.5 \\
\hline BOD & $\mathrm{mgL}^{-1}$ & 0.2 & 1.7 & $0.96 \pm 0.52$ & - & \\
\hline COD & $\mathrm{mgL}^{-1}$ & 65 & 95 & $48.8 \pm 10.50$ & - & - \\
\hline Turbidity & NTU & 5 & 7 & $6 \pm 0.700$ & Max-5 & Max-5 \\
\hline Salinity & $\mathrm{mgL}^{-1}$ & 0.01 & 0.04 & $0.02 \pm 0.01$ & - & - \\
\hline T. Hardness & $\mathrm{mgL}^{-1}$ & 30 & 32.5 & $32 \pm 0.89$ & Max-500 & Max-500 \\
\hline T. Alkalinity & $\mathrm{mgL}^{-1}$ & 20.80 & 25.19 & $22.70 \pm 1.53$ & - & - \\
\hline T. Acidity & $\mathrm{mgL}^{-1}$ & 8 & 14 & $10.6 \pm 2.10$ & - & - \\
\hline $\mathrm{CO}_{2}$ & $\mathrm{mgL}^{-1}$ & 6 & 12 & $8.7 \pm 2.0$ & - & - \\
\hline Chloride $\left(\mathrm{Cl}^{-}\right)$ & $\mathrm{mgL}^{-1}$ & 4 & 8 & $6.00 \pm 0.50$ & Max-500 & Max-250 \\
\hline Sulphate $\left(\mathrm{SO}_{4}{ }^{2-}\right)$ & $\mathrm{mgL}^{-1}$ & 3 & 8 & $6.3 \pm 0.17$ & Max-400 & Max-400 \\
\hline Phosphate $\left(\mathrm{PO}_{4}^{3-}\right)$ & $\mathrm{mgL}^{-1}$ & 0.01 & 0.41 & $0.25 \pm 0.14$ & Max-6 & - \\
\hline Nitrate $\left(\mathrm{NO}_{3}^{-}-\mathrm{N}\right)$ & $\mathrm{mgL}^{-1}$ & 0.5 & 0.9 & $0.7 \pm 0.13$ & Max-4.5 & Max-10 \\
\hline Nitrite $\left(\mathrm{NO}_{2}^{-}-\mathrm{N}\right)$ & $\mathrm{mgL}^{-1}$ & 2.25 & 4.05 & $3.15 \pm 1.15$ & Nil & 0.50 \\
\hline Arsenic (As) & $\mathrm{mgL}^{-1}$ & 0.00 & 0.03 & $0.02 \pm 0.00$ & 0.05 & 0.01 \\
\hline Cadmium $(\mathrm{Cd})$ & $\mathrm{mgL}^{-1}$ & 0.00 & 0.00 & $0.00 \pm 0.00$ & 0.005 & 0.003 \\
\hline Cromium (Cr) & $\mathrm{mgL}^{-1}$ & 0.00 & 0.04 & $0.02 \pm 0.01$ & 0.05 & 0.05 \\
\hline Copper $(\mathrm{Cu})$ & $\mathrm{mgL}^{-1}$ & 0.00 & 0.16 & $0.07 \pm 0.03$ & 1.0 & 0.05 \\
\hline Cobalt (Co) & $\mathrm{mgL}^{-1}$ & 0.00 & 0.00 & $0.00 \pm 0.00$ & 0.003 & 0.005 \\
\hline Iron $(\mathrm{Fe})$ & $\mathrm{mgL}^{-1}$ & 0.16 & 0.32 & $0.21 \pm 0.14$ & $0.3-1.0$ & 0.3 \\
\hline Lead $(\mathrm{Pb})$ & $\mathrm{mgL}^{-1}$ & 0.00 & 0.00 & $0.00 \pm 0.00$ & Max-0.1 & Max-0.1 \\
\hline Manganese (Mn) & $\mathrm{mgL}^{-1}$ & 0.00 & 0.74 & $0.31 \pm 0.18$ & 0.01 & 1.5 \\
\hline Nickel (Ni) & $\mathrm{mgL}^{-1}$ & 0.00 & 0.6 & $0.25 \pm 0.14$ & $0.3-1.0$ & $0.1-1.0$ \\
\hline Zinc $(\mathrm{Zn})$ & $\mathrm{mgL}^{-1}$ & 2.25 & 4.05 & $3.051 \pm 1.03$ & 5.0 & 3.0 \\
\hline
\end{tabular}

Note: Min $=$ Minimum, Max $=$ Maximum. BSTI $=$ Bangladesh Standards and Testing Institute. WHO $=$ World Health Organization. ${ }^{*}=$ The measure of precision is the standard deviation (s).

\section{CONCLUSION:}

From this research work it is clear that in every parameter of effluents, the value is much higher than acceptable limit which is so much frightening for us. So, the properly untreated waste water mainly heavy metals are deposited in our bodies through food cycle and we have to face kidney diseases, lever diseases and even cancer. Different water quality parameters of the Kaptai Lake studied (Table 2, $3 \&$ \& ) show that $\mathrm{pH}$ was found in the alkaline range (7.7-8.8), and water was found in acidity $\left(2.8-4.60 \mathrm{mgL}^{-1}\right)$. EC (95.6$\left.115.20 \mu \mathrm{Scm}^{-1}\right)$, and TDS (47.80-57.600 $\left.\mathrm{mgL}^{-1}\right)$. DO was found $\left(7.60-8.20 \mathrm{mgL}^{-1}\right)$, and Alkalinity of the 
Kaptai Lake $\left(42.90-51.00 \mathrm{mgL}^{-1}\right)$ is mainly for carbonates and bicarbonates as phenolphthalein alkalinity was not detected in any of the samples. Water was found in turbidity as (1.00-2.5 NTU). Total hardness was found in the range (35.00-42.00 $\left.\mathrm{mLL}^{-1}\right)$. Chloride (7.00-12.00 $\left.\mathrm{mgL}^{-1}\right)$, BOD $\left(0.2-0.5 \mathrm{mgL}^{-1}\right)$, nitrite-N (2.25-6.75) and nitrate-N (0.70-1.3 $\left.\mathrm{mgL}^{-1}\right)$. Phosphate-P (0.010-0.052 $\left.\mathrm{mgL}^{-1}\right)$ and sulfate-S (1.1$\left.3.00 \mathrm{mgL}^{-1}\right)$ were also found within the permissible limit. Iron (0.1-1.7 $\left.\mathrm{mg} \mathrm{L}^{-1}\right)$ and manganese (0.00$0.07 \mathrm{mg} \mathrm{L}^{-1}$ ) were found within the limit. Different water quality parameters of the Foy's Lake studied (Table 4 \& 6) show that $\mathrm{pH}$ was found in the alkaline range (7.5-8.0) and water was found in acidity (8.00 $\left.14.00 \mathrm{mgL}^{-1}\right)$. EC (66-72.80 $\left.\mu \mathrm{Scm}^{-1}\right)$ and TDS (33.00$\left.72.80 \mathrm{mgL}^{-1}\right)$. DO was found $\left(7.70-8.10 \mathrm{mgL}^{-1}\right)$. Alkalinity of the Foy's Lake $\left(20.80-25.19 \mathrm{mgL}^{-1}\right)$ is mainly for carbonates and bicarbonates as phenolphthalein alkalinity was not detected in any of the samples. Water was found in turbidity as (5.00$7.00 \mathrm{NTU})$. Total hardness was found in the range (30.00-32.50 mgL ${ }^{-1}$ ). Chloride (4.00 - $8.00 \mathrm{mgL}^{-1}$ ), BOD (0.2-1.7 mgL-1), nitrite-N (2.25-4.05) and nitrate-N (0.50 - $\left.0.9 \mathrm{mgL}^{-1}\right)$. Phosphate-P (0.01 - 0.51 $\left.\mathrm{mgL}^{-1}\right)$ and sulfate-S $\left(3.0-8.00 \mathrm{mgL}^{-1}\right)$ were also found within the permissible limit. Iron $(0.16-0.32 \mathrm{mg}$ $\mathrm{L}^{-1}$ ) and manganese $\left(0.00-0.74 \mathrm{mgL}^{-1}\right)$ were found within the limit. So, formal and informal approaches of education might be adopted through regional media, seminars, celebrations, workshops, walks, and student competitions to aware the people regarding the activity of environment degradation. Then our environment, our eco system will free from pollution.

\section{ACKNOWLEDGEMENT:}

Many thanks to the co-authors supported with proper assistance and help for data analysis to conduct successful research work.

\section{CONFLICTS OF INTEREST:}

The authors announced no prospective conflicts of the interest with respect to the present research work.

\section{REFERENCES:}

1) Ahmed M. J., M. R. and Haque T.M.A., (2010). "Physicochemical assessment of surface \& groundwater resources of Comilla region of Bangladesh", International J.of Chemical \& Environ. Engineering. 1, 45-55.

2) Ahmed T, Uddin ME, Alam MK, Moniruzzaman M, Saha B, Sufian A, Alam M. G, Hossain I. (2018). Evaluation of Microbial and Physiochemical Properties of Three Selected Lakes Water in Dhaka City, Bangladesh. Scholars Academic J. of Biosciences. 6(2): 230-238.

3) Aris Z., M. H. Abdullah, A. Ahmed and K. K. Woong, (2007). International Journal of Environmental Science and Technology, 4(4), 441.

4) Diersing Nancy (2009). Water Quality: Frequently asked Questions. Florida Brook National Marine Sanctuary, Key West, FL.

5) EWD, (2009). "Earth's water distribution (EWD)". United States Geological Survey. Retrieved

6) Flanagan, S.M., Montgomery, D.L. and Ayotte, J.D., (2001). U.S. Geological Survey Water Resources Investigation Report 014042, p. 12

7) FRSS, (2012), Fisheries Statistical Yearbook of Bangladesh, Fisheries Resources Survey System (FRSS), Department of Fisheries, Bangladesh, 28: 46

8) Henriksen A. and Posch, (2001). Steady-state models for calculating critical loads of acidity for surface waters. Water Air Soil Pollut. Focus, 1, 375-398.

9) Hirsch R.M., (2007). NAWQA program, USGS.

http://nh.water.usgs.gov/pubpg.comment.htm

10) Hossain MM, Majumder KA, Islam $M$, and Nayeem AA. (2019). Study on Ambient Particulate Matter (PM2.5) with Different Mode of Transportation in Dhaka City, Bangladesh. Am. J. Pure Appl. Sci., 1(4), 1219. https://doi.org/10.34104/ajpab.019.0191219

11) Kabir, A.K. and M.N. Naser, (2011). Physicochemical aspects of Chandbill Oxbow Lake of Meherpur, Bangladesh. Dhaka University J. of Biological Sciences. 20(1): 31-39. 
12) Kramer Kj, Jak RG, Van Hattum B, Hooftman RN, Zwolsman JJ , (2004). "Copper toxicity in relation to surface water-dissolved organic matter: biological effects to Daphnia magna", Environ Toxicol Chem., 23(12), 2971-80.

13) Lasat, M.M. (2002). Phytoextraction of Toxic Metals: A Review of Biological Mechanisms. Journal of Environmental Quality, 31, 109120. http://dx.doi.org/10.2134/jeq2002.0109

14) Majagi S. H., Vijayakumar K. and Vasanthkaumar B. (2008). Environ Monit Assess, 138, 273-279.

15) Salt, D.E., Blaylock, M. Kumar, P.B.A.N, Dushenkov, V., Ensley, B.D., Chet, L. and Raskin, L. (1995) Phytoremediation: A Novel Strategy for the Removal of Toxic Metals from the Environment Using Plants. Biotechnology, 13, 468-474. http://dx.doi.org/10.1038/nbt0595-4688
16) Shukla, P., Preeti and A. Singh, (2013). A seasonal variation of plankton population of Maheshara Lake in Gorakhpur, India. World J. of Zoology, 8(1): 9-16

17) Upadhyay, K., Mishra P. and Gupta A.K. (2010). Studies on the physicochemical status of two ponds at Varanasi and Bhadohi under biotic stress. Plant Arch. 10 (2): 691-693.

18) WHO, (1984). Guidelines for Drinking Water Quality- Health Criteria and other Supporting Information. World Health Organization, Geneva, Switzerland

19) WHO, (1991). Revision of the WHO Guidelines for Water Quality Report of the First Review Group Meeting on Inorganics, (Netherlands) world Health Organization Geneva, WHO/PEP, 18

20) WHO, (2004). Rapid Needs Assessment for Water, WHO, Regional Office for South-East Asia, New Delhi, India.

Citation: Rubel M, Chowdhury DA, Ahmed MJU, and Uddin MH. (2019). Physico-chemical characterization of kaptai lake and foy's lake water quality parameters in Chittagong, Bangladesh. Am. J. Pure Appl. Sci., 1(6), 4958. https://doi.org/10.34104/ajpab.019.01949058 (c) Ð 